\title{
Avaliação da demanda de intervenções de enfermagem em terapia intensiva segundo o Nursing Activities Score (NAS)
}

\author{
Evaluation of the demand for nursing interventions in intensive therapy according to the Nursing \\ Activities Score (NAS) \\ Evaluación de la demanda para intervenciones de enfermería en terapia intensiva según el \\ Nursins Activities Score (NAS)
}

Paola Marsura Verreschi de Oliveira1*, Karisa Santiago Nakahata², Isaac Rosa Marques².

\begin{abstract}
RESUMO
Objetivo: Identificar a frequência das intervenções de enfermagem mais prevalentes em UTI e identificar a possibilidade de o planejamento da assistência acontecer de forma mais real e objetiva com avaliação de demanda pelo Nursing Activities Score (NAS) com avaliação do tempo dispendido e dimensionamento adequado. Verificar a influência de variáveis sobre o NAS e analisar a mortalidade pela correlação do NAS com o Simplified Acute Physiology Score 3 (SAPS 3). Correlacionar o NAS com o Therapeutic intervention scoring system-28 (TISS-28). Método: Estudo prospectivo com o método da coleta manual de dados e análise de prontuário, com um "n" de 179 pacientes. Os dados foram compilados e analisados do ponto de vista da estatística descritiva. Resultados: O NAS médio foi de 71,43. Não houve influência das variáveis idade, tempo de permanência e procedência, a variável destino pós-alta apresentou correlação positiva considerando o desfecho óbito. Correlação positiva do TISS-28 com exceção do domínio de atividades básicas. O SAPS 3 mostrou correlação positiva onde o NAS eleva-se nos pacientes que foram a óbito. Inadequação quanto ao dimensionamento das unidades estudadas. Conclusão: O NAS é um instrumento que abrange o exigido para avaliação de demanda de cuidados, se traduzindo em uma ferramenta válida de gestão.
\end{abstract}

Palavras chaves: Enfermagem de cuidados críticos, Dimensionamento de pessoal, Carga de trabalho, Cuidados de enfermagem, Cuidados críticos.

\begin{abstract}
Objective: To identify the frequency of the most prevalent nursing interventions in the ICU and to identify the possibility of planning the care more realistically and objectively with the Nursing Activities Score (NAS) assessment of the time spent and adequate design. To verify the influence of variables on the NAS and analyze the mortality by the correlation of the NAS with the Simplified Acute Physiology Score 3 (SAPS 3). Correlate the NAS with the Therapeutic intervention scoring system-28 (TISS-28). Method: Prospective study using manual data collection and chart analysis, with an " $n$ " of 179 patients. Data were compiled and analyzed from the point of view of descriptive statistics. Results: The mean NAS was 71.43. There was no influence of the variables age, length of stay and origin, the post-discharge destination variable presented a positive correlation considering the death outcome. Positive correlation of TISS-28 with the exception of the domain of basic activities. SAPS 3 showed a positive correlation where NAS rises in patients who died. Inadequacy in the dimensioning of the studied units. Conclusion: The NAS is an instrument that covers what is required to assess the demand for care, translating into a valid management tool.
\end{abstract}

Keywords: Critical Care Nursing, Personnel Downsizing, workload, Nursing care, Critical care.

\footnotetext{
${ }^{1}$ Enfermeira discente do programa de pós-graduação na modalidade de residência multiprofissional em Emergências Clínicas e Trauma da Universidade de Santo Amaro. *E-mail: paolamarsura@gmail.com ${ }^{2}$ Prof. Ms. Docente na Universidade de Santo Amaro no programa de pós-graduação na modalidade de residência multiprofissional em Emergências Clínicas e Trauma.
} 


\section{RESUMEN}

Objetivo: Identificar la frecuencia de las intervenciones de enfermería más prevalentes en UTI e identificar la posibilidad de que la planificación de la asistencia ocurra de forma más real y objetiva con evaluación de demanda por el Nursing Activity Score (NAS) con evaluación del tiempo gastado y dimensionamiento adecuado. Verificar la influencia de variables sobre el NAS y analizar la mortalidad por la correlación del NAS con el Simplified Acute Physiology Score 3 (SAPS 3). Correlacionar el NAS con el enfoque terapéutico del sistema-28 (TISS-28). Método: Estudio prospectivo con el método de la recolección manual de datos y análisis de prontuario, con un " $n$ " de 179 pacientes. Los datos fueron compilados y analizados desde el punto de vista estadístico descriptivo. Resultados: El NAS medio fue de 71,43. No hubo influencia de las variables edad, tiempo de permanencia y procedencia, la variable destino post-alta presentó correlación positiva considerando el desenlace muerto. Correlación positiva del TISS-28 con excepción del dominio de actividades básicas. EI SAPS 3 mostró correlación positiva donde el NAS se eleva en los pacientes que murieron. Inadecuación en cuanto al dimensionamiento de las unidades estudiadas. Conclusión: El NAS es un instrumento que abarca lo necesario para la evaluación de la demanda de cuidados, que se traduce en una herramienta válida de gestión.

Palabras claves: Enfermería de cuidados críticos; reducción de personal, carga de trabajo; atención de enfermería; cuidados críticos.

\section{INTRODUÇÃO}

Define-se Unidade de Terapia Intensiva (UTI) como uma área crítica destinada à pacientes graves ou em situações de risco, que necessitam de atenção profissional especializada de forma contínua (Ministério da Saúde, 2010). A evolução da UTI alterou todo o cenário do cuidado, as mudanças no perfil dos pacientes e das unidades críticas requerem uma melhor qualificação e quantificação dos profissionais que atuam na UTI (CONISHI RMAY, GAIZINSKI RP 2017).

As UTIs são consideradas como as unidades que proporcionam a assistência mais complexa, sofisticada e onerosa do sistema de saúde, pois concentram alta especificidade de recursos humanos e tecnológicos (QUEIJO AF, 2002). Para realizar o dimensionamento de uma UTI deve-se considerar duas vertentes: uma equipe reduzida pode impactar na queda da eficiência da assistência e uma equipe superdimensionada pode implicar em um alto custo para a instituição (GONÇALVES LA, PADILHA KG, 2007).

A equipe de enfermagem representa o percentual quantitativo e orçamentário mais significativo em uma UTI (GAIDZINSKI RR, 1998). Os indicadores de demanda de cuidados são considerados como requisitos mínimos para assegurar a qualidade da assistência prestada, o uso destes indicadores favorece 0 planejamento da assistência (QUEIJO AF, PADILHA KG, 2009; FEITOSA MC, et al., 2012).

Atualmente o instrumento de medida de força de trabalho de enfermagem mais utilizado em UTI é o Nursing Activities Score (NAS). O NAS foi validado e traduzido para o português em 2002, ele é o resultado da modificação do Therapeutic Intervention Scoring System-28 (TISS-28). O NAS contempla 80,8\% das atividades de enfermagem, superando o TISS-28 com sua abrangência de 43,3\%. (QUEIJO AF, 2002; QUEIJO AF, PADILHA KG, 2009).

O NAS é um instrumento que pontua a necessidade de cuidados de enfermagem em 24 horas, é composto por 7 grandes categorias, subdividas em 23 itens. A cada item é atribuído uma pontuação que varia de $1,2 \mathrm{a}$ 32,0. A soma do seu resultado final representa a porcentagem de tempo gasto pelo profissional de enfermagem, cada um ponto do NAS equivale a 14,4 minutos do tempo gasto pela enfermagem (FEITOSA MC, et al., 2012; FERREIRA PC, et al 2014).

Para compreender melhor a relação da pontuação do NAS com a mortalidade do paciente é realizada a análise do índice prognóstico Simplified Acute Physiology Score (SAPS 3). O sistema prognóstico SAPS 3 é composto de 20 variáveis que visam estabelecer um índice preditivo de mortalidade para pacientes admitidos em UTI. (JÚNIOR JMS, et al., 2010)

A utilização destes instrumentos avalia de forma objetiva a condição clínica do paciente bem como suas reais necessidades de cuidado, sendo indispensáveis para assegurar um planejamento da assistência que 
se traduza em sinônimo de qualidade (QUEIJO AF, 2002). Para analisar o dimensionamento das unidades estudas é realizado o cálculo de dimensionamento de pessoal proposto por Gaidznsk (GAIDZINSKI RR, 1998), conforme a resolução do Conselho Federal de Enfermagem (COFEN) n 543/2017 (Manual do Nursing Activties Score - NAS, 2010)

Sendo assim, o objetivo deste estudo foi de identificar a frequência das intervenções de enfermagem mais prevalentes em uma UTI e identificar a possibilidade do planejamento da assistência acontecer de forma mais real e objetiva.

\section{MÉTODO}

Trata-se de uma pesquisa do tipo epidemiológica observacional descritiva de originalidade primária de seguimento transversal e direcionalidade prospectiva.

A pesquisa foi realizada nas unidades de terapia intensiva adulto de um hospital público estadual de médio porte localizado na região sul da cidade de São Paulo. Uma unidade é composta por 10 leitos sendo um deles destinado para pacientes em isolamento e a outra unidade é composta por 14 leitos. Para fins de nomenclatura a unidade de 14 leitos foi denominada como UTI NORTE e a unidade de 10 leitos de UTI SUL.

A população estudada compreendeu os pacientes admitidos nas duas unidades no período de janeiro a março de 2017, considerando uma média mensal de trânsito de 345 pacientes nas unidades por uma amostra de conveniência e aplicando a amostragem probabilística (MAROTTI J, et al., 2008) com um erro amostral de 5\% (nível de confiança de 95\%) e percentual máximo de 40\% (considerando a heterogeneidade da amostra), chegou-se ao "n" amostral de 179 casos. Considerando a razão da proporcionalidade das 179 aplicações, 81 ocorreram na UTI SUL e 98 na UTI NORTE. A fim de retratar de forma fiel o cenário do estudo foi utilizado na pesquisa a reaplicação do instrumento em um mesmo paciente ao longo de sua permanência.

Os critérios de inclusão foram: todos os pacientes maiores de 16 anos de ambos os sexos e como critérios de exclusão aqueles que não completavam 24 horas na unidade.

Para realização da pesquisa foi solicitado e concedido pelo Hospital Geral do Grajaú uma declaração de coparticipante. A pesquisa foi submetida à apreciação da Comissão de Ética e Pesquisa da Universidade de Santo Amaro e ao Comitê de Ética e Pesquisa do Hospital Geral do Grajaú. Obtendo aprovação pelo CAAE do CEP UNISA n 61031016.0.0000.0081 e CAAE do CEP do Hospital Geral do Grajaú coparticipante n 61031016.0.3001.5447.

Foi elaborado e submetido o Termo de Compromisso e Confidencialidade, no qual a pesquisadora assegura a guarda, cuidado e sigilo com o prontuário do paciente. Aos pacientes e/ou familiares participantes da pesquisa foi explicado e entregue o Termo de Consentimento Livre e Esclarecido - (TCLE). Os termos foram elaborados conforme a resolução 466 de 12 de dezembro de 2012.

A coleta de dados procedeu-se a partir de um instrumento construído pela pesquisadora que compilava o NAS em sua íntegra conforme a versão validada em 2002 (QUEIJO AF, 2002) acrescido de um cabeçalho que possibilitou a coleta de dados pertinentes a identificação do paciente, sua internação e seu desfecho clínico, o instrumento também continha o TISS-28 na sua integra e um campo para coleta de dados referente a aplicação do SAPS 3 e sua probabilidade de óbito.

Após a coleta os dados foram compilados em uma planilha do Microsoft@ Excel Professional Plus 2016 e analisados por meio da estatística descritiva (BELLO A, 2001) e pela aplicação de testes paramétricos e não paramétricos no software $R \circledast$ versão 3.4.3, onde para avaliar a correlação entre os escores (pontuações totais e pontuações por domínio), foi considerado o coeficiente de correlação de Spearman e o teste para verificação se tal coeficientes é diferente de zero. O coeficiente de correlação é uma medida que varia entre -1 e 1 , sendo que quanto mais próximo de 0 , menor a correlação entre duas variáveis e quanto mais próximo de 1(-1), maior a correlação positiva (negativa) entre as variáveis de interesse.

Entende-se por correlação positiva, a tendência crescente de uma variável conforme os valores da outra 
também aumentam, já por correlação negativa, a tendência decrescente de uma das variáveis enquanto o valor da outra aumenta.

Foi considerado um nível de significância de $5 \%$, ou seja, quando o p-valor é inferior a 0,05 , temos indícios da existência de correlação não nula, a qual pode ser fraca, moderada ou forte de acordo com o valor do coeficiente estimado. Para explicar a correlação entre os escores totais, foram obtidos modelos de regressão linear simples.

\section{RESULTADOS}

Das 179 aplicações do instrumento a pesquisa obteve 116 pacientes distintos e 63 reaplicações. A amostra foi caracterizada por $53,07 \%$ de homens, $42,46 \%$ de idosos, encontrou-se um número considerável de jovens adultos $24,14 \%$ na faixa etária de 46 a 55 anos. $63,69 \%$ dos pacientes permaneceram até 10 dias internados na UTI.

Quanto a procedência do paciente crítico, 43,02\% eram provenientes do serviço de emergência e a maior razão de internação foi de etiologia clínica com $86,59 \%$. Entre as causas a maior demanda é cardiovascular (26,82\%), seguida de infecção e sepse (18,44\%). Quanto ao desfecho clínico, as 179 coletas representavam 116 pacientes distintos, onde 38 (32,76\%) evoluíram a óbito. O NAS médio foi de $71,43 \%$ nas duas unidades. Na UTI NORTE a média do NAS foi de $66,84 \%$ e na UTI SUL de $76,98 \%$. Ao considerar os 14,4 minutos para cada ponto do NAS obteve-se uma média de assistência de 16 horas e 4 minutos na UTI NORTE e de 18 horas e 47 minutos na UTI SUL.

A Tabela 1 apresenta os coeficientes de correlação entre as pontuações dos subdomínios dos escores NAS e TISS-28. Observa-se que apenas o subdomínio de atividades básicas apresentou uma correlação fraca $(\mathrm{cor}=0,249)$.

Tabela 1 - Valores estimados do coeficiente de correlação de Spearman para as pontuações dos subdomínios do NAS e TISS, São Paulo - SP, 2017.

\begin{tabular}{|c|c|c|c|c|c|c|}
\hline $\begin{array}{l}\text { Atividades } \\
\text { Básicas (TISS) }\end{array}$ & $\begin{array}{l}\text { Atividades } \\
\text { Básicas } \\
\text { (NAS) }\end{array}$ & $\begin{array}{c}\text { Suporte } \\
\text { ventilatório } \\
\text { (NAS) }\end{array}$ & $\begin{array}{c}\text { Suporte } \\
\text { cardiovascular } \\
\text { (NAS) }\end{array}$ & $\begin{array}{l}\text { Suporte } \\
\text { renal } \\
\text { (NAS) }\end{array}$ & $\begin{array}{l}\text { Suporte } \\
\text { metabólico } \\
\text { (NAS) }\end{array}$ & $\begin{array}{l}\text { Intervenções } \\
\text { específicas } \\
\text { (NAS) }\end{array}$ \\
\hline C. Correlação & 0,249 & 0,195 & 0,224 & 0,005 & 0,182 & 0,270 \\
\hline $\mathrm{p}$-valor & 0,001 & 0,009 & 0,003 & 0,944 & 0,015 & 0,000 \\
\hline \multicolumn{7}{|l|}{$\begin{array}{l}\text { Suporte } \\
\text { ventilatório } \\
\text { (TISS) }\end{array}$} \\
\hline C. Correlação & 0,486 & 0,990 & 0,383 & 0,064 & 0,800 & 0,275 \\
\hline p-valor & 0,000 & 0,000 & 0,000 & 0,396 & 0,000 & 0,000 \\
\hline \multicolumn{7}{|l|}{$\begin{array}{l}\text { Suporte } \\
\text { cardiovascular } \\
\text { (TISS) }\end{array}$} \\
\hline C. Correlação & 0,288 & 0,396 & 0,911 & 0,201 & 0,392 & 0,340 \\
\hline $\mathrm{p}$-valor & 0,000 & 0,000 & 0,000 & 0,007 & 0,000 & 0,000 \\
\hline \multicolumn{7}{|l|}{$\begin{array}{l}\text { Suporte Renal } \\
\text { (TISS) }\end{array}$} \\
\hline C. Correlação & $-0,003$ & 0,053 & 0,146 & 0,866 & 0,108 & 0,210 \\
\hline$p$-valor & 0,969 & 0,482 & 0,051 & 0,000 & 0,148 & 0,005 \\
\hline \multicolumn{7}{|l|}{$\begin{array}{l}\text { Suporte } \\
\text { metabólico } \\
\text { (TISS) }\end{array}$} \\
\hline C. Correlação & 0,427 & 0,777 & 0,345 & 0,141 & 0,992 & 0,275 \\
\hline p-valor & 0,000 & 0,000 & 0,000 & 0,061 & 0,000 & 0,000 \\
\hline \multicolumn{7}{|l|}{$\begin{array}{l}\text { Intervenções } \\
\text { específicas } \\
\text { (TISS) }\end{array}$} \\
\hline C. Correlação & 0,085 & 0,224 & 0,264 & 0,100 & 0,213 & 0,941 \\
\hline p-valor & 0,260 & 0,003 & 0,000 & 0,183 & 0,004 & 0,000 \\
\hline
\end{tabular}

Fonte: Elaborado pela autora, dados de janeiro a março de 2017. 
A variáveis idade e tempo de permanência ao serem analisadas pela correlação de Pearson apresentaram uma correlação nula com o NAS. Para a variável idade, foi obtido um $\mathrm{p}=0,095827$ e, para tempo de permanência, um $\mathrm{p}=0,014675$. Quanto a variável procedência observa-se que não há discrepância entre os valores mínimos e máximos atingidos com o NAS mantendo uma mediana de 58,85 a 74,15. A maior diferença se apresenta nos valores obtidos de pacientes provenientes de outro hospital, porém esta variável também possui a menor razão de procedência (1,12\%), que representa cerca de dois pacientes na amostra de 179 . Conclui-se que o valor final do NAS independe do setor de procedência.

Quanto a variável destino após a alta, foi observado uma tendência entre os valores médios com exceção daquelas que o desfecho clínico ou destino após a alta tenha sido o óbito, observou-se uma mediana de 55 a 67,5 quando considerado o destino em sim e uma mediana de 55 a 83 quando considerado o desfecho clínico óbito. O que se conclui da análise desta variável é o que o que influencia no valor do NAS não é o destino pós alta per se é, sim, a condição clínica do paciente. A análise da correlação do NAS com o TISS28 apresentou um coeficiente de correlação de $0,632(p=0,001)$ o que indica uma correlação linear moderada e positiva entre as pontuações. Os resultados do modelo de regressão linear para as pontuações totais dos escores NAS e TISS-28 indicam que um aumento de uma unidade no valor da pontuação total do TISS-28 leva a um aumento médio de 1,51 para pontuação do NAS.

Quanto à correlação do escore NAS com o índice prognóstico SAPS 3, o coeficiente de correlação entre os escores totais NAS e a probabilidade SAPS 3 foi de $0,394(p=0,001)$, indicando uma correlação fraca a moderada. O gráfico 1 apresenta a dispersão dos valores das pontuações $O$ coeficiente do modelo de regressão linear para a pontuação total do escore NAS e o valor de probabilidade de óbito do SAPS 3 indica que um aumento de uma unidade no valor da probabilidade leva a um aumento médio de 0,29 na pontuação do NAS.

Gráfico 1 - Dispersão para pontuações totais Probabilidade de óbito (SAPS 3) e NAS, São Paulo SP, 2017.

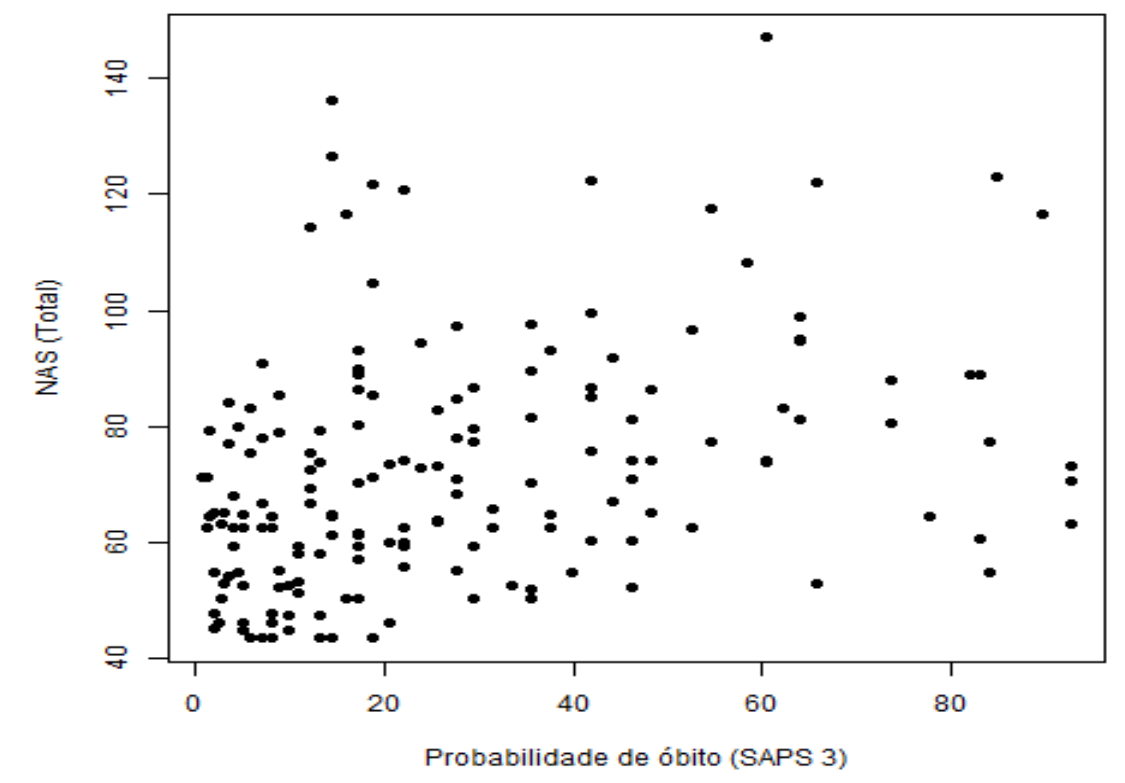

Fonte: Elaborado pela autora, dados de janeiro a março de 2017.

Para a análise dos óbitos foi considerado o maior valor do NAS entre todas as medidas de coleta de cada paciente, tendo como banco de dados 116 pacientes e 38 óbitos (32,76\%). A Tabela 2 apresenta medidas descritivas para os escores NAS (maior valor) e probabilidade de óbito de acordo com o óbito ou não dos indivíduos. 
Tabela 2 - Medidas descritivas para probabilidade de óbito (SAPS3) e NAS de acordo com óbito ou não dos pacientes (Teste Mann-Whitney), São Paulo - SP, 2017.

\begin{tabular}{lcccc}
\hline & Não & Óbito & Sim & p-valor \\
\hline Probabilidade de óbito SAPS 3 & Média (DP) & $16,15(14,79)$ & $44,62(26,11)$ & 0,001 \\
& Mediana (IIQ) & $10,41(5,11 ; 22,15)$ & $41,92(20,89 ; 63,59)$ & \\
NAS & Média (DP) & $64,58(14,63)$ & $94,66(22,42)$ & 0,001 \\
& Mediana (IIQ) & $63,00(53,05 ; 71,40)$ & $89,35(78,45 ; 115,85)$ &
\end{tabular}

${ }^{*}$ DP: Desvio padrão, IIQ: Intervalo interquartílico (quartis $25 \%$ e $75 \%$ ).

Fonte: Elaborado pela autora, dados de janeiro a março de 2017.

A Figura 1 apresenta a projeção para o maior valor do NAS (esquerda) e probabilidade de óbito (direita). Observa-se que os valores médios e medianos para probabilidade de óbito são maiores nos pacientes que foram a óbito, sendo identificada diferença significativa $(p=0,001)$, o que também pode ser observado no boxplot, onde a distribuição da probabilidade de óbito (representada pelo "quadrado do gráfico") é consideravelmente superior para pacientes que foram a óbito. Analogamente os pacientes que foram a óbito têm maiores valores médios e medianos do NAS do que os pacientes em que o óbito não foi registrado.

Figura 1 - Boxplot para NAS (esquerda) e Probabilidade de óbito (direita) de acordo com o óbito, São Paulo - SP, 2017.
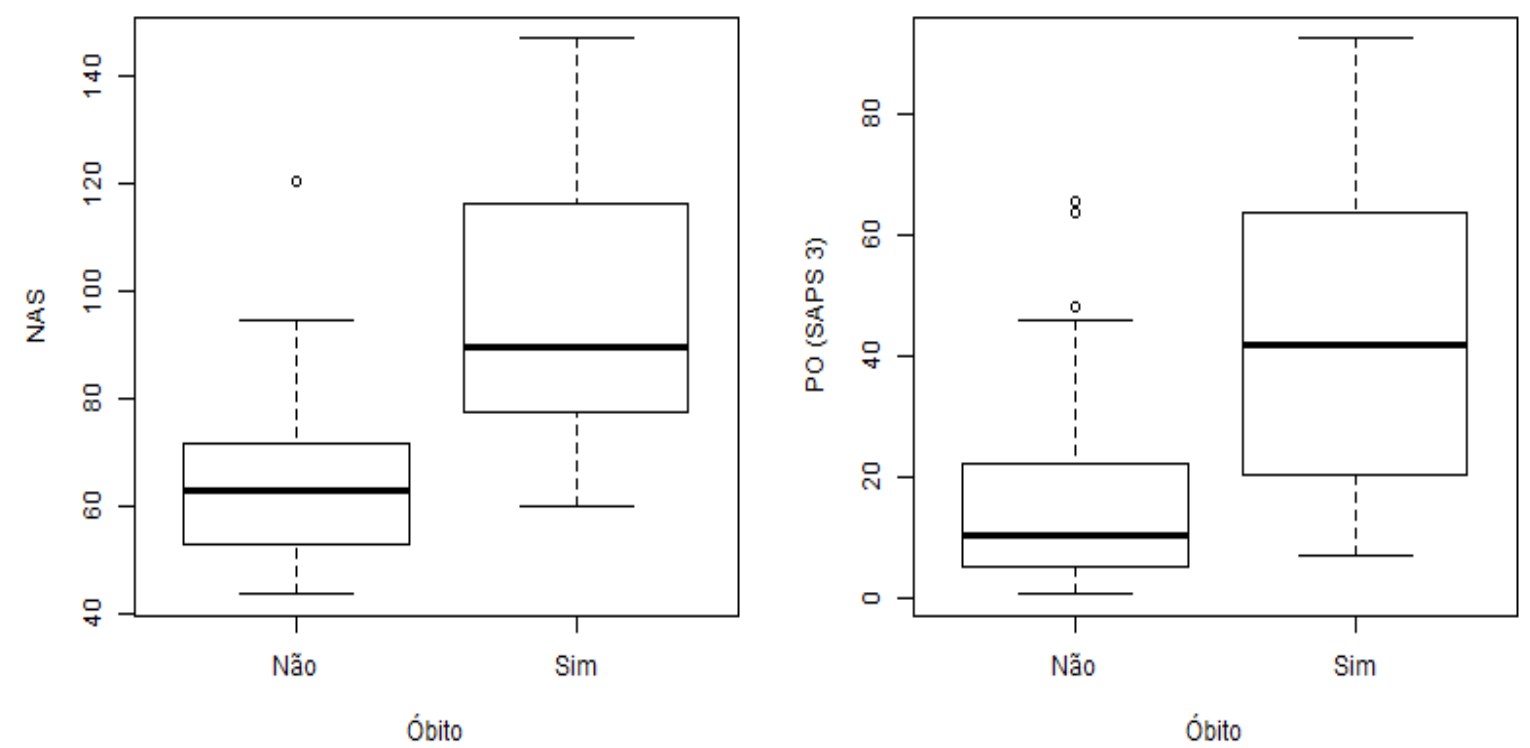

Fonte: Elaborado pela autora, dados de janeiro a março de 2017.

Adicionalmente, o gráfico 2 apresenta a dispersão entre maior NAS e probabilidade de óbito no qual os pontos são coloridos de acordo com o óbito ou não. Esse gráfico facilita a visualização do fato de que pacientes que vão a óbito tendem a ter maiores valores em ambos os escores, porém ainda existem casos em que o escore NAS é baixo com uma probabilidade de óbito alta e vice-versa, o que justifica a correlação fraca calculada anteriormente. 
Gráfico 2 - Dispersão para pontuações totais Probabilidade de óbito (SAPS 3) e maior NAS, SP 2017.

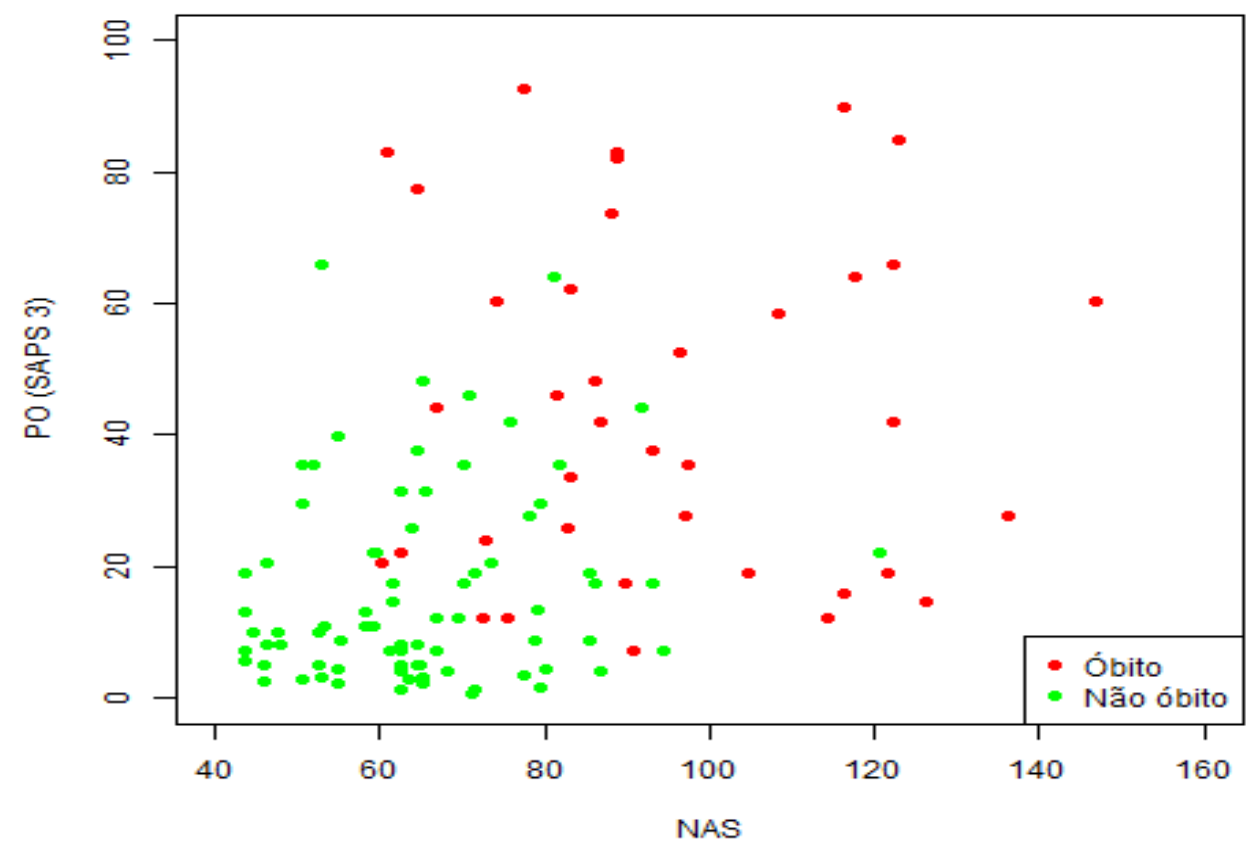

Fonte: Elaborado pela autora, dados de janeiro a março de 2017.

Considerando a resolução n 543/2017 do COFEN aplicou-se o cálculo de dimensionamento proposto por Gaidzinski. Na UTI NORTE foi considerado o valor mínimo estabelecido pela resolução de 17,9 horas e para UTI SUL o valor de tempo estimado pela aplicação do escore NAS de 18,47 horas. Para realização do cálculo de (GAIDZINSKI RR, 1998) considerou-se uma taxa de ocupação de $100 \%$, um total de horas de enfermagem (THE) de 250,6 para UTI NORTE e 184,7 para UTI SUL, o índice de segurança técnica (IST) foi o mínimo estabelecido pela resolução de $15 \%$ e para a Constante de Marinho (KM) o valor de 0,2236. Assim obteve-se que o quantitativo mínimo de pessoal para UTI NORTE deveria ser de 56 colaboradores e para UTI SUL de 41 colaboradores. Estabelecendo o mínimo de 52\% de enfermeiros propostos pela resolução n 543/2017 o quadro de pessoal deveria ser idealmente composto por 29 enfermeiros e 27 técnicos de enfermagem na UTI NORTE e por 21 enfermeiros e 19 técnicos de enfermagem na UTI SUL. Identifica-se um déficit de 22 colaboradores para UTI NORTE e 7 para UTI SUL.

\section{DISCUSSÃO}

Os estudos brasileiros que utilizaram o NAS no ambiente de terapia intensiva apontam que a maioria dos pacientes eram de homens e idosos (CONISHI RMAY, GAIZINSKI RP, 2007; QUEIJO AF, 2002; CIAMPONE JT, et al, 2006; FERREIRA PC, 2014; LIMA MKF, et al., 2008; GONÇALVES LA, et al., 2006; NOGUEIRA LS, et al, 2017). A porcentagem de $24,14 \%$ de jovens adultos pode ser explicada pelo fato da instituição hospitalar do estudo ser referência em emergências clínicas e trauma e estar localizada em uma região carente da cidade de São Paulo. A população da zona sul de São Paulo segundo o censo levantado pelo IBGE (Instituto Brasileiro de Geografia e Estatística) e trabalhados pela GISA (Gerência de Geoprocessamento e Informações Socioambientais) estimam uma população de cerca de 11.253.471 onde há um número estimado de 1.409 .996 jovens adultos entre 44 a 55 anos e uma estimativa de 1.885 .570 idosos acima de 55 anos (Áreas de Abrangência das Unidades de Saúde no Município de São Paulo - Censo 2010 - Versão 2015/2017). 
Um estudo (PAGLIARINI FC, 2012) realizado em um hospital de ensino da cidade de Ribeirão Preto do estado de São Paulo obteve um tempo médio de internação de 12,76 dias ( $D P=10,30$ ). Outro estudo (NOGUEIRA LS, et al., 2007) realizado nesta mesma instituição hospitalar identificou um tempo de permanência de 9 a 10 dias. Este estudo de 2007 apontou que 73,3\% dos pacientes advinham do pronto socorro e $22,3 \%$ do centro cirúrgico, o que é corroborado pelos estudos brasileiros que apontam que em sua maioria grande parte da demanda dos pacientes que necessitam de UTI são provenientes destes setores (QUEIJO AF, 2002; GONÇALVES LA, et al., 2006; NOGUEIRA LS, et al., 2007; PAGLIARINI FC, 2012; CIAMPONE JT, et al, 2006).

Quando à causa de internação clínica, o resultado encontrado no presente estudo está de acordo com outras pesquisas brasileiras (QUEIJO AF, 2002; BULECHECK GM, et al., 2010). A taxa de mortalidade encontrada neste estudo foi de $32,76 \%$. Em estudo (NOGUEIRA LS, et al, 2007) realizado na mesma instituição da pesquisa em 2007, a taxa de mortalidade foi de 29,7\%. Na literatura é possível identificar taxas de mortalidade com valores médios de $31,43 \%$ a $38 \%$ em estudos realizados no estado de São Paulo. Fora do estado de São Paulo a literatura apresenta valores entre 10,6 a 27,5\% (FERREIRA PC, et al., 2014; FERREIRA PC, 2014; PAGLIARINI FC, 2012; LEITE IRL, et al., 2012; CIAMPONE JT, et al, 2006; BULECHECK GM, et al., 2010).

A comparação da taxa de mortalidade com estudos internacionais mostra que a taxa de mortalidade encontrada em território nacional encontra-se bastante elevada, em média foi identificado uma mortalidade de 9,2\% em estudos estrangeiros (CARMONA-MFJ, et al., 2013; STAFSETH SK, et al., 2011).

O valor médio do NAS encontrado neste estudo foi de 71,43\%. Estudos realizados no Brasil em UTI adulto, unidade de terapia semi-intensiva e UTI neonatal obtiveram um NAS com valor médio entre $45,1 \%$ a $91,1 \%$ (QUEIJO AF, 2002; GONÇALVES LA, PADILHA KG, 2007; CIAMPONE JT, et al., 2006; CASTILHO LS, 2011; ALTAFIN JAM, et al., 2014). Um estudo (FERREIRA PC, et al., 2014) realizado em 2014 sobre a aplicação do NAS, compilou evidências, apontando que a média do NAS foi superior a $50 \%$, ficando em $60 \%$ dos estudos entre $60-70 \%$ com apenas quatro estudos com média acima de $70 \%$, no qual destes, três foram realizados no Brasil.

A literatura (FERREIRA PC, 2014; PAGLIARINI FC, 2012; LEITE IRL, et al., 2012; CIAMPONE JT, et al., 2010; ALTAFIN JAM, et al, 2014) brasileira aponta que não há influência da variável idade sobre o NAS conforme identificado nesta pesquisa. Porém, os estudos analisados apenas um vai de acordo ao encontrado nesta pesquisa sobre a não influência do variável tempo de permanência sobre o NAS (PAGLIARINI FC, 2012). Os demais estudos apontam que, quanto maior o tempo de permanência na unidade, maior é o valor do NAS (CONISHI RMAY, GAIZINSKI RP, 2007; GONÇALVES LA, PADILHA KG, 2007; GONÇALVES LA, et al., 2006; PADILHA KG, et al., 2008; QUEIJO AF, et al., 2013; INOUE KC, et al., 2011; PANUNTO MR, GUIRARDELLO EB, 2012).

Acredita-se que este resultado possa ser justificado pelo fato do hospital onde foi realizado o estudo ser um centro hospitalar de porta aberta localizado na periferia de São Paulo, onde os pacientes já são admitidos em estado críticos até mesmo por falta de acesso a rede primária de serviços de saúde, chegando assim a unidade hospitalar já em estado avançado de doença. Sendo admitidos na UTI já com um NAS elevado no início da internação hospitalar. Um estudo de Pagliarini corrobora com o achado desta pesquisa, concluindo que o valor final do NAS independe do setor de procedência do paciente (PAGLIARINI FC, 2012).

Em relação a variável destino após a alta, os resultados do presente estudo estão de acordo com os encontrados na literatura, nos quais é apontado que o que influencia no valor do NAS não é o destino pós alta em si, e sim a condição clínica do paciente. Pacientes que têm como desfecho clínico o óbito, tendem a ter um NAS mais elevado, tanto em decorrência da gravidade clínica do mesmo quanto da demanda de cuidados que o óbito onera para a equipe de enfermagem (FERREIRA PC, et al, 2014; GONÇALVES LA, et al., 2006; PAGLIARINI FC, 2012; LEITE IRL, et al., 2012; CASTILHO LS, 2011; PADILHA KG, et al., 2008; ALTAFIN JAM, et al, 2014; QUEIJO AF, et al., 2013). 
Ao realizar a validação e adaptação transcultural do NAS em 2002, Queijo (QUEIJO AF, 2002) correlacionou os escores do NAS e do TISS-28 estabelecendo uma associação de $67 \%$ entre eles, identificando que a cada um ponto de aumento do TISS-28, a pontuação do NAS tende a aumentar em torno de 2,59 vezes. Apesar de ser identificado nesta pesquisa uma correlação moderada, a tendência de aumento do NAS conforme o TISS-28 foi menor do que o proposto por Queijo (QUEIJO AF, 2002), com um aumento médio de 1,51 vezes. Os achados desta pesquisa corroboram o estudo de Queijo (QUEIJO AF, 2002), identificando que o domínio com menor índice de correlação foi o de atividades básicas, resultado este esperado vez que é descrito na literatura que este domínio foi fator determinante para a reconstrução do instrumento de avaliação.

Não foi identificado na literatura algum estudo que correlaciona diretamente o NAS com o SAPS 3. Porém, no estudo realizado em 2002 por Queijo (QUEIJO AF, 2002), houve relação positiva entre o valor encontrado de SAPS 2 e o NAS e em outro estudo realizado em 2007 na instituição desta pesquisa, observou-se uma relação positiva entre o NAS e o índice de gravidade APACHE, índice este que verifica o grau de disfunção orgânica, traduzindo sua gravidade e maior probabilidade de óbito em um valor numérico assim como o SAPS 3. O SAPS 2 foi apresentado em 1993 e compreendia 12 variáveis fisiológicas, idade, tipo de admissão e presença de doença crônica já o SAPS 3 atualmente compreende 20 variáveis representadas por escore fisiológico agudo e avaliação do estado prévio, visando estabelecer índice preditivo de mortalidade para pacientes admitidos em unidades de terapia intensiva.

Os estudos apontam que o subdimensionamento e a sobrecarga de trabalho para a equipe de enfermagem geram custos incalculáveis relacionados a vidas perdidas, lesões irrecuperáveis e estresse dos trabalhadores. (QUEIJO AF, 2002; FERREIRA PC, et al., 2014; FERREIRA PC, 2014; GONÇALVES LA, et al., 2006). Em um estudo (INOUE KC, MATSUDA L, 2010) realizado no Paraná, foi concluído que a aplicação do NAS ajustados às recomendações da Resolução COFEN n 543/2017 podem contribuir para o dimensionamento adequado em uma UTI, favorecendo assim condições de trabalho adequadas, qualidade e segurança. Outro estudo (FERREIRA PC, et al., 2014) apresenta evidências sobre o NAS, no qual foi apontado que em 16 estudos brasileiros houve inadequação no que diz respeito ao dimensionamento de pessoal de enfermagem. Outro estudo (CONISHI RMAY, GAIZINSKI RP, 2007) apresentou resultados positivos em relação do dimensionamento de pessoal em consonância com o proposto pelo NAS.

Considerando o cenário identificado nesta pesquisa observa-se que é necessário uma adequação não somente no que diz respeito ao quantitativo de pessoal mas também em relação à categoria profissional dos colaboradores, uma vez que o número mínimo de enfermeiros em cada unidade (17,65\%) é bem distante do mínimo previsto pela legislação vigente e o contingente de profissionais auxiliares de enfermagem $(41,18 \%$ na UTI NORTE e 50\% na UTI SUL). Esse dado destoa do que é sugerido pela legislação, ressaltando que é claro, no que diz respeito que à assistência a pacientes que requeiram cuidados intensivos, tal assistência deve ser prestada por enfermeiros e técnicos de enfermagem.

\section{CONCLUSÃO}

Pode-se afirmar que o uso do NAS permite um planejamento da assistência mais real e objetivo propiciando ao enfermeiro uma ferramenta para realizar um dimensionamento justo e seguro. O perfil clínico encontrado nos pacientes desta pesquisa é condizente com a literatura brasileira sobre pacientes críticos em unidade de terapia intensiva. A taxa de mortalidade encontrada apesar de elevada é considerada esperada quando comparada a estudos nacionais. A demanda de cuidados do NAS em relação a horas de trabalho é similar ao encontrado em estudos brasileiros, com uma demanda aproximadamente de 17 horas e 15 minutos de assistência com um NAS médio de $71,43 \%$. O NAS nesta pesquisa não sofreu influência das variáveis idade, tempo de permanência e procedência. Quanto ao destino pós-alta o NAS sofreu influência quando o destino foi relacionado com o desfecho clínico do paciente, no caso, o óbito. A correlação com o TISS-28 mostrou que há forte relação entre seus domínios com exceção ao domínio que diz respeito a atividades básicas. A correlação do NAS com o SAPS 3 apontou uma tendência que mostra um aumento do NAS 
conforme o aumento do SAPS 3 quando associado a ocorrência de óbito. O óbito eleva o valor do NAS em decorrência da gravidade a qual o paciente se encontrava e a alta demanda de cuidados pós morte. Foi identificado um déficit no que diz respeito a quantitativo de pessoal e a categoria profissional.

\section{REFERÊNCIAS}

1. ALTAFIN JAM, et al. Nursing Activities Score e carga de trabalho em unidade de terapia intensiva de hospital universitário. Rev. Bras. Ter. Inten. 2014 set; 26(3): 292-8.

2. Associação Brasileira de Enfermagem em Terapia Intensiva. Manual do Nursing Activties Score - NAS. São Paulo; 2014. Disponível em: http://www.abenti.org.br/site/wp-content/uploads/2014/04/curso-de-NAS.pdf. Acesso em 27 ago 2017.

3. BELLO A. Análise descritiva de dados. In: LoBiondo-Wood G, Haber J. Pesquisa em enfermagem - métodos, avaliação crítica e utilização. $4^{\mathrm{a}}$ ed. Rio de Janeiro: Guanabara-Koogan; 2001. p. 200-10.

4. BULECHECK GM, et al. Classificação das Intervenções de Enfermagem - NIC. 5ed. Rio de Janeiro: Elsevier; 2010.

5. CARMONA-MFJ, et al. Carga de trabajo en tres grupos de pacientes de UCI Española según el Nursing Activities Score. Rev. Esc. Enferm. USP 2013 mar; 47(2): 335-40.

6. CASTILHO LS. Nursing Activities Score (NAS): carga de trabalho de enfermagem em Unidade de Terapia Intensiva Neonatal Dissertação - Universidade Federal do Rio Grande do Sul, Escola de Enfermagem, Porto Alegre, 2011.

7. CIAMPONE JT, et al. Necessidade de cuidados de enfermagem e intervenções terapêutic as em UTI: estudo comparativo entre pacientes idosos e não idosos. Acta Paul. Enferm. 2006 jan; 19(1): 28-35.

8. CIAMPONE JT, et al. Necessidade de cuidados de enfermagem e intervenções terapêuticas em UTI: estudo comparativo entre pacientes idosos e não idosos. Acta Paul. Enferm. 2006 jan; 19(1): 28-35.

9. CONISHI RMAY, GAIZINSKI RP. Nursing Activities Score (NAS) como instrumento para medir carga de trabalho de enfermagem em UTI adulto. Rev. Esc. Enferm. USP. 2007 maio; 41(3): 346-54

10. FEITOSA MC, et al. Demanda de enfermagem a pacientes intensivos: NAS. Esc Anna Nery. 2012 out; 16 (4):682-8.

11. FERREIRA PC, et al. Dimensionamento de enfermagem em Unidade de Terapia Intensiva: evidências sobre o Nursing Activities Score. Rev. Rene. 2014 set; 15(5): 888-97.

12. FERREIRA PC. Nursing Activities Score: avaliação da carga de trabalho de enfermagem em Unidade de Terapia Intensiva Adulto [dissertação]. Natal: Universidade Federal do Rio Grande do Norte; 2014.

13. GAIDZINSKI RR. Dimensionamento de pessoal de enfermagem em instituições hospitalares Tese - Escola de Enfermagem, Universidade de São Paulo, São Paulo, 1998.

14. GONÇALVES LA, et al. Necessidades de cuidados de enfermagem em terapia intensiva: evolução diária dos pacientes segundo o Nursing Activities Score (NAS). Rev. Bras. Enferm. 2006 jan; 59(1): 56-60.

15. GONÇALVES LA, PADILHA KG. Fatores associados à carga de trabalho de enfermagem em Unidade de Terapia Intensiva. Rev. Esc. Enferm. USP. 2007 dez; 41(4): 645-52.

16. INOUE KC, et al. Nursing Activities Score (NAS): carga de trabalho de enfermagem em UTI e fatores associados. Cienc. Cuid. Saúde. 2011 jan: 10(1):134-40.

17. INOUE KC, MATSUDA LM. Dimensionamento de pessoal de enfermagem em Unidade de Terapia Intensiva para adultos. Acta Paul. Enferm. 2010 jun; 23(3): 379-84.

18. JÚNIOR JMS, et al. Aplicabilidade do escore fisiológico agudo simplificado (SAPS 3) em hospitais brasileiros. Rev. Bras. Anestesiol. 2010 jan; 60(1): 20-31

19. LEITE IRL, et al. Nursing Activities Score e demanda de trabalho de enfermagem em terapia intensiva. Acta Paul. Enferm. 2012 dez; 25(6): 837-43.

20. LIMA MKF, et al. Aplicação do Nursing Activities Score em pacientes de alta dependência de enfermagem. Texto contexto - Enferm. $2008 \mathrm{dez} ; 17(4)$ : 638-46.

21. MAROTTI J, et al. Amostragem em pesquisa clínica: tamanho da amostra. Rev. Odontol Univer. Cidade São Paulo. 2008 maioago; 20(2): 186-94.

22. Ministério da Saúde (BR). Agência Nacional de Vigilância Sanitária. Resolução № 7, de 24 de fevereiro de 2010. Dispõe sobre os requisitos mínimos para funcionamento de Unidades de Terapia Intensiva e dá outras providências. Diário Oficial da União, Brasília, 25 fev. 2010. Seção 1, p. 48-58

23. NOGUEIRA LS, et al. Nursing Activities Score: comparação com o Índice APACHE II e a mortalidade em pacientes admitidos em unidade de terapia intensiva. Rev. Bras. Ter. Intens. 2007 set; 19(3): 327-30.

24. PADILHA KG, et al. Nursing Activities Score in the intensive care unit: analysis of the related factors. Intensive Crit. Care Nurs. 2008 jun; 24(3): 197-204.

25. PAGLIARINI FC. Identificação da carga de trabalho de enfermagem segundo o Nursing Activities Score (NAS) em unidade de terapia intensiva de um hospital de ensino. Dissertação - Universidade de São Paulo, Escola de Enfermagem de Ribeirão Preto, Ribeirão Preto, 2012

26. PANUNTO MR, GUIRARDELLO EB. Carga de trabalho de enfermagem em Unidade de Terapia Intensiva de um hospital de ensino. Acta Paul. Enferm. 2012 jan; 25(1): 96-101.

27. Prefeitura do Município de São Paulo. Áreas de Abrangência das Unidades de Saúde no Município de São Paulo - Censo 2010 Versão 2015/2017 atualizado em 09 de agosto de 2017. Disponível em: http://www.prefeitura.sp.gov.br/cidade/secretarias/saude/epidemiologia e informacao/informacoes socioambientais/index.php?p $=8452$. Acesso em 07 ago 2016.

28. QUEIJO AF, et al. Nursing workload in neurological intensive care units: Cross-sectional study. Intensive Crit. Care Nurs. 2013; (29):112-6.

29. QUEIJO AF, PADILHA KG. Nursing Activities Score (NAS): adaptação transcultural e validação para a língua portuguesa. Rev. Esc. Enferm. USP. 2009 dez; 43(esp): 1018-25.

30. QUEIJO AF. Tradução para o português e validação de um instrumento de medida de carga de trabalho de enfermagem em Unidade de Terapia Intensiva: Nursing Activities Score (NAS) Dissertação - Escola de Enfermagem. Universidade de São Paulo, São Paulo, 2002.

31. STAFSETH SK, et al. The characterization of workloads and nursing staff allocation in intensive care units: A descriptive study using the Nursing Activities Score for the first time in Norway. Int. Crit. Care Nurs. 2011: (27):290-4. 